\title{
RESEARCH ON OPTIMAL CONTROL METHOD OF ACTIVE NOISE REDUCTION OF ACTIVE NOISE CANCELLING HEADPHONES COMBINED WITH NOISE REDUCTION OF PHONON MATERIALS IN HIGH VOLTAGE CONVERTER STATION
}

\author{
Yanhe Sun ${ }^{1}$, Jinan Liu' ${ }^{1}$, Ruiguo Chen ${ }^{2}$, Yanlong Li ${ }^{2}$, Zhifei Niu ${ }^{1}$ \\ ${ }^{1}$ State Grid Liaoning Maintenance Company, Shenyang, Liaoning 110003, China \\ ${ }^{2}$ State Grid Liaoning Electric Power Supply Co., Ltd., Shenyang, Liaoning, 110003, China \\ E-mails: yanh1975@yeah.net,jnanliu@yeah.net,rgor66@126.com \\ yanlli@163.com,zfn zf@yeah.net
}

\begin{abstract}
The magnetostriction phenomenon of the converter transformer radiates high-decibel noise, and the noise is mainly low-frequency, and the noise penetration ability is strong and the propagation distance is long. Low-frequency noise can reach the ear bones easily, easily irritating the human nerves, causing symptoms such as rapid heartbeat and elevated blood pressure, which is more serious than medium and high-frequency noise damage, which seriously affects the work and life of the station staff. Active noise canceling headphones are difficult to achieve good noise reduction for high frequency noise. Based on this, this paper puts forward a new idea of incorporating active noise canceling headphones into phononic materials, and carries out preliminary verification by experimental method, and obtains better noise reduction effect. This paper is designed to promote the active noise reduction headphones to provide better reference for noise reduction, and has certain practical significance.
\end{abstract}

Keywords: Converter Transformer; Active Noise Canceling Earphone; Phonon Material.

\section{Introduction}

With the continuous development of the social economy, people pay more and more attention to environmental issues. As an important environmental pollution, noise pollution has received more and more attention [1]. Long-term exposure to high noise conditions, people's hearing and physical health will be seriously affected, even the general noise will have different degrees of impact on people's normal life and work. Therefore, for the high-voltage converter station, the noise generated will inevitably have a great negative impact on the health of the staff. Therefore, noise control is a key task that needs to be emphasized and implemented in this field [2-3]. Strategically speaking, noise control can start from three aspects: noise source, noise propagation path and noise receiver. The traditional noise control technology is mainly based on the acoustic control method of noise. The main technical methods include sound absorption processing, sound insulation processing, use of muffler and so on. These noise control methods mainly consume energy through the interaction of noise sound waves with acoustic materials or acoustic structures, thereby achieving the purpose of noise reduction. It is a passive or passive control method and can be called "passive" noise control [4]. In general, passive noise control is effective for medium and high frequency noise, while control of low frequency noise is not effective due to structural and space constraints. To this end, active noise control technology has emerged [5,6]. Among them, active noise control refers to a method of artificially generating secondary noise to cancel the original noise, which can better reduce the noise of low frequency noise. In view of the characteristics of active noise reduction and passive noise reduction, this paper will combine active noise canceling headphones with phononic materials, so that noise canceling headphones can better reduce noise for low, medium and high frequency noise. The effect is to provide protection for the staff of the high-voltage converter station.

\section{Adaptive Active Noise Reduction Principle and System Structure Analysis}

- Adaptive active noise reduction principle

There are two main methods of noise reduction in terms of noise reduction, one is Active Noise 
Control (ANC), and the other is Passive Noise Control (PNC). Among them, passive noise reduction mainly uses the sound absorption and isolation of materials to isolate sound and block the outside sound from entering the current subject. However, this isolation method is only suitable for high-frequency noise, and the isolation effect for low-frequency noise is not ideal, and there is even the possibility of aggravating noise or reducing the desired signal energy. It shows the limitations of the passive noise reduction method. The ANC uses a set of noise reduction control circuit to generate noise with the opposite phase of noise and energy and frequency through the calculation and adjustment of the controller to reduce noise. Specifically as shown in Figure 1.

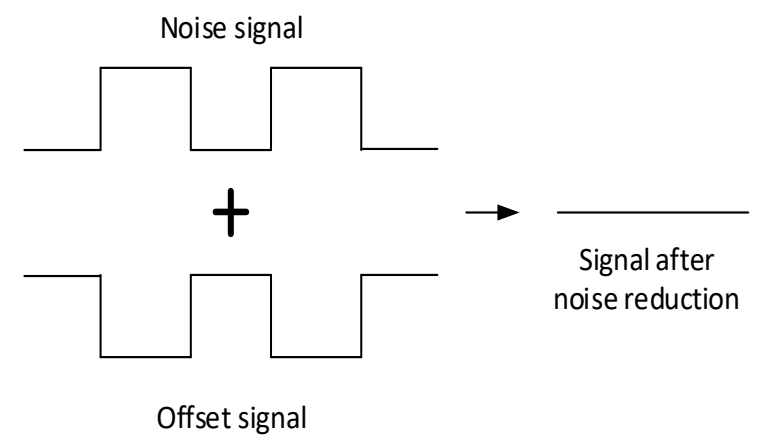

Figure 1: Schematic diagram of active noise reduction

Active noise reduction In an ideal state, although the noise can be completely canceled, in reality, the signal after noise reduction has a certain volatility, and this volatility depends on the adaptive adjustment capability of the noise reduction control. The concept of active noise reduction is not difficult to understand, but adaptive controllers that achieve perfect noise reduction are difficult to design. At present, the active noise reduction system is divided into feedforward noise reduction, feedback noise reduction, and front and feedback combined noise reduction. The specific analysis is as follows.

\section{- Structural analysis of active noise reduction system}

(1) Feedforward active noise reduction control system

The feedforward active noise reduction control system (open loop noise control) requires two microphones for sound collection [7, 8]. The structure of the system is shown in Figure 2. The reference microphone collects the noise signal and transmits it to the controller. The calculation of the conversion by the controller is performed by using the speaker output to cancel the phase of the noise signal opposite to the noise signal. Then, the residual noise input after noise reduction is collected by the error microphone to the controller for calculation, and the controller coefficient is updated, so that it can be iterated.

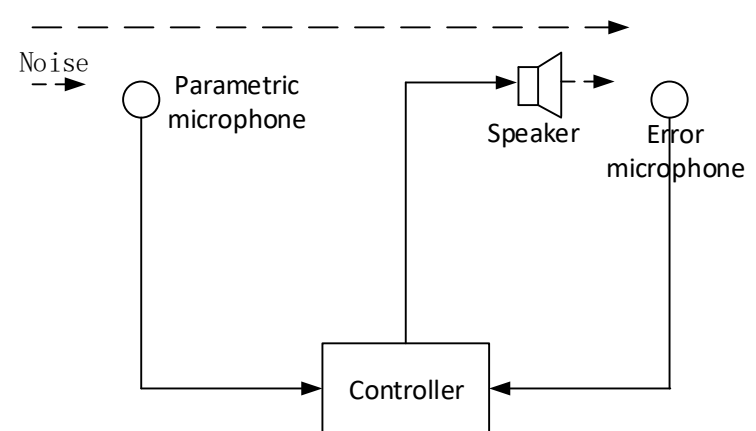

Figure 2: Structure of feedforward active noise reduction system

Feedforward main advantage is to reduce the noise of the reference microphone directly collected the original noise, the microphone and speaker unit separated design, thus ensuring the sound wave effect on the reference microphone, speaker but feedforward circuit in signal transmission between the speakers and microphone error process, there have been many times of reflection, therefore, the final output results may be unpredictable.

(2) Feedback active noise reduction control system

The feedback active noise reduction control system, also known as the closed-loop noise reduction control system, has only one error microphone [9]. The signal collected by microphone is input to the controller to update the filter weight coefficient. However, because the system is a closed loop system, it is easy to generate self-excited oscillation, which leads to the instability of the system and becomes a disadvantage in the design of feedback noise reduction circuit.

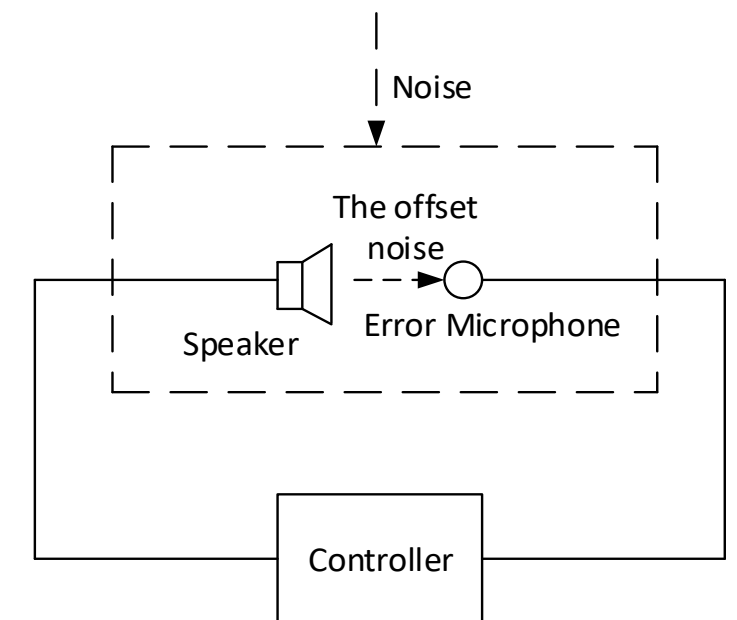

Figure 3: Structure of feedback active noise reduction system

(3) Front and feedback combined active noise reduction control system

Figure 4 shows the combined noise reduction system structure of front and feedback, which has two controllers, namely, feedforward controller and 
feedback controller [10]. Feedback controller and feedforward controller jointly determine the output of secondary channels, and the structure under combination is decoupled. The advantage of decoupling structure is that feedforward and feedback can be independently designed and recombined.

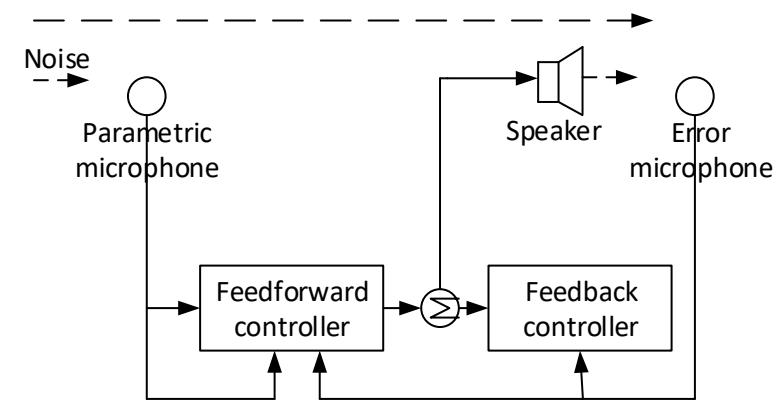

Figure 4: Feedforward and feedback combined noise reduction system

\section{Principle of LMS Adaptive Filtering Algorithm}

Due to the characteristics of the primary sound source and the surrounding environment changing at any time, as well as the amplitude and phase characteristics of the sound signal emitted by the secondary sound source, the controller needs to be adjusted adaptively to achieve better noise reduction effect. In order to achieve this goal, an adaptive algorithm is added in the data processing. The adaptive filtering algorithm includes adaptive algorithm and filter, in which the weight coefficient of the filter is adjusted by the adaptive algorithm. Compared with other filters, the stability of finite impulse response filter (FIR) is better. The algorithm adopts Least mean square (LMS) algorithm. Figure 5 shows the adaptive filtering structure, where $x(k)$ is the reference signal, which is the signal emitted by the primary sound source in the experiment. $d(k)$ is the expected signal; $e(k)$ is the error signal, that is, the signal collected from the secondary sound source; $y(k)$ is the output signal, representing the final output signal of the secondary sound source. Suppose the filter order is $L$ and the weight coefficient is $w_{l}(k)(l=1,2, \cdots, L)$, then the output signal $y(k)$ can be expressed as:

$$
y(k)=\sum_{l=1}^{L} w_{l}(k) x(n-l+1)
$$

Reference signal $x(k)$ and filter weight coefficient $W$ can be expressed as:

$$
X(k)=[x(k), x(k-1), \cdots x(k-L+1)]^{T}
$$

$$
W(k)=[w(k), w(k-1), \cdots w(k-L+1)]^{T}
$$

The output signal $y(k)$ can be obtained from equation (1) - equation (3)

$$
y(k)=X^{T}(k) W(k)=W^{T}(k) X(k)
$$

Then, error signal $e(k)$ at time $k$ is:

$$
e(k)=d(k)-y(k)=d(k)-W^{T}(k) X(k)
$$

If $u$ is the convergence coefficient, namely the step size factor, then the adaptive iteration formula of the weight coefficient is:

$$
W(k+1)=W(k)+2 \mu e(k) X(k)
$$

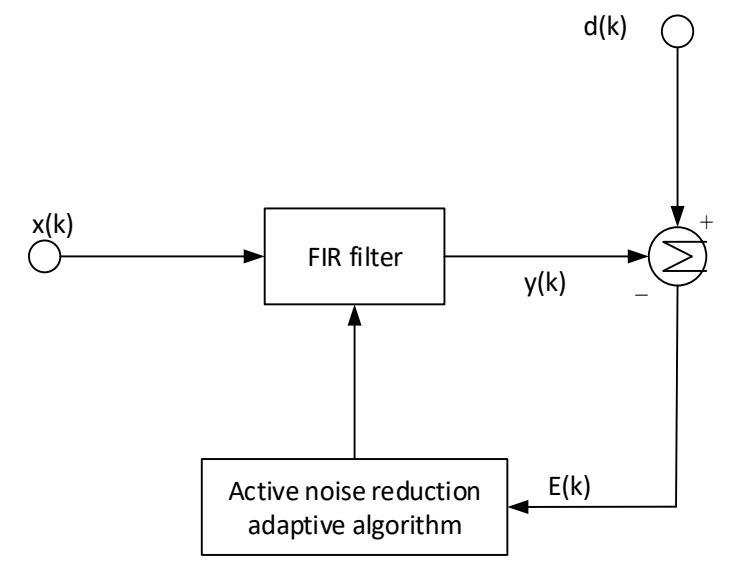

Figure 5: Adaptive filtering structure

\section{Performance Test of LMS Feedforward Adaptive Active Noise-Canceling Earphone}

Through the above theoretical analysis, we have a certain understanding of the structure of active noise reduction system and LMS algorithm. Based on this, this paper decides to test the performance of LMS feedforward adaptive active noise-canceling earphone, aiming to verify the active noise-canceling effect of active noise-canceling earphone in high voltage converter station.

The details are as follows:

\section{- Equipment determination and test conditions}

In order to reduce the interference brought by the external environment, this paper carried out the test under the environment of soundproof room, the necessary equipment is: preamplifier/audio distributor, host, power amplifier, B\&K tester, highpower speaker. 
The test conditions are: the order length of the filter is 64 , the convergence factor is 0.001 , the reference signal is a mixed signal of audio signal and white noise, the test frequency is $20-20 \mathrm{kHz}$, and the test voltage is $179 \mathrm{mV}$.

\section{- $\quad$ Test steps and results}

During the test, the host generates noise signal. After the power amplifier amplifies, the high-power horn sends out the simulated noise signal. The active anti-noise earphone is installed on the artificial ear. The artificial ear picks up the sound pressure signal of the earphone, amplifies it through the pre-power amplifier, and outputs it to the host. Finally, according to the frequency response curve and distortion curve obtained by the host, the advantages and disadvantages of LMS feedforward adaptive active noise reduction earphone are analyzed. Where, frequency response, referred to as frequency response, measures the ability of electro-acoustic devices to uniformly reproduce signals in high, medium and low frequency bands. The evaluation of the frequency response curve mainly has two aspects. First, the range should be as wide as possible. Second, the curve within the frequency range as flat as possible to avoid excessive fluctuations.

Total harmonic distortion is the extra harmonic component of the output signal over the input signal, usually expressed as a percentage. The total harmonic distortion is below $1 \%$, which is indistinguishable to the ear. The smaller the total harmonic distortion, the purer the sound. The corresponding frequency curve of the measured active noise-canceling earphone is shown in Figure 6. As can be seen from the Figure, this active noisecanceling earphone can reproduce the sound in the range of 20-20 KHZ with a flat curve and good performance.

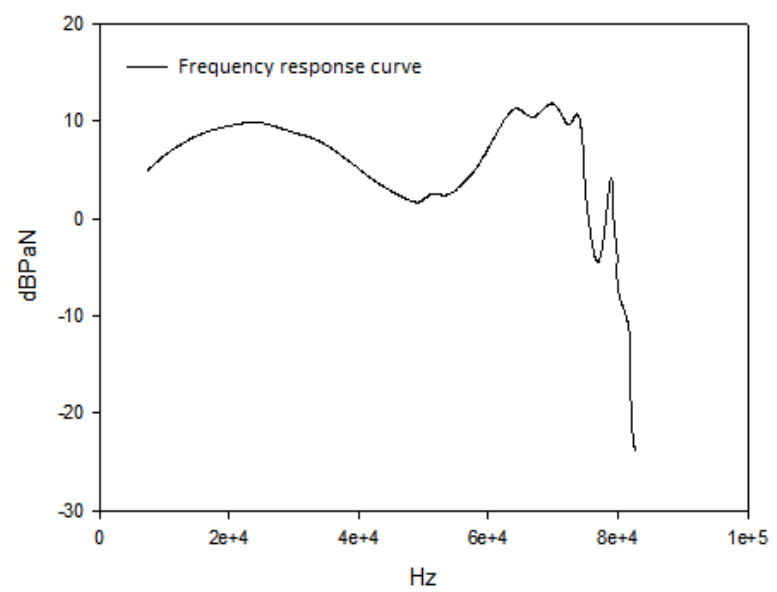

Figure 6: Frequency response curve

The total harmonic distortion curve of the active noise-canceling headphones is shown in Figure 7.

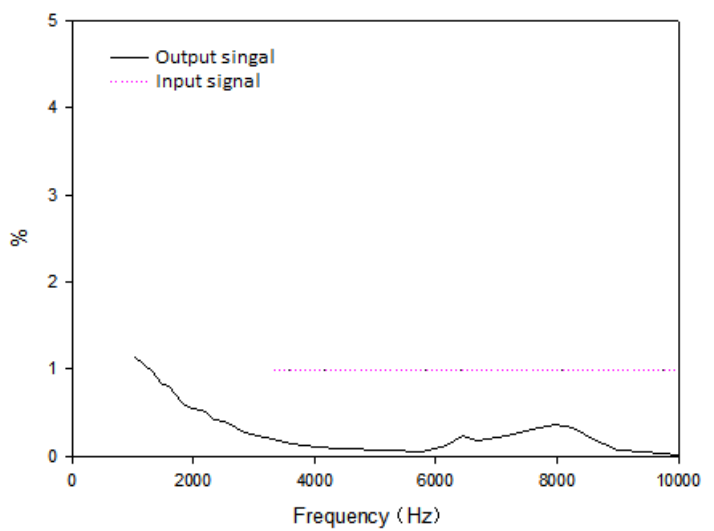

Figure 7: Distortion curve

It can be seen from the Figure that the active noise-canceling earphone is in the effective sound frequency range of $20-20 \mathrm{khz}$, with distortion degree less than $1 \%$ and good performance.

Finally, the sound pressure of the reference signal is respectively output. The sound pressure of the output signal without starting the noise reduction circuit (passive noise reduction) and the sound pressure spectrum of the output signal after starting the noise reduction circuit (active noise reduction) are compared, as shown in Figure 8.

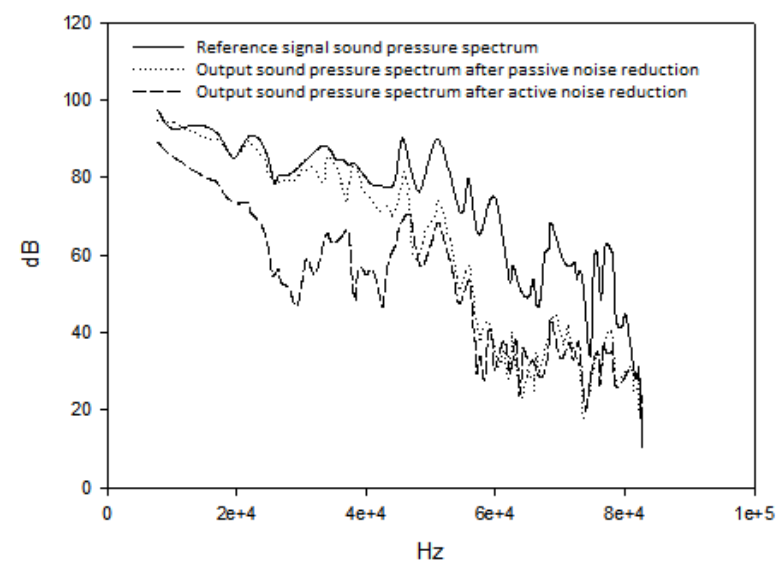

Figure 8: Comparison of noise reduction effect

It can be seen that the noise reduction effect of active noise-canceling headphones for low and medium frequency signals is significantly better than that of passive noise-canceling headphones, while for high frequency signals, active noise-canceling headphones have no obvious advantage.

\section{Analysis of Noise Reduction Effect after Combining Phonon Crystal Materials}

According to the above detection, it can be known that active noise-canceling earphones can achieve better noise canceling effect in low and medium frequency signals, while the advantages of noise 
canceling in high frequency signals are not obvious. In order to improve the noise reduction effect of active noise-canceling headphone in high frequency signal, phonon crystal material is integrated in this paper. Phononic crystal is a new type of functional material, has been developing rapidly in recent years, the phononic crystal is a kind of complex acoustic material, its structure consists of several elastic material or periodic arrangement of soundabsorbing material, and when the elastic wave frequency within the scope of phononic crystals bandgap, elastic wave propagation will be inhibited or prohibited, so as to achieve the effect of noise reduction, this characteristic for vibration noise reduction provides a new method and train of thought.

- Phonon crystal material selection and experimental parameter setting

In order to improve the noise reduction, the idea of embedding phonon crystal into active noise reduction system is proposed, which is based on the combination of passive technology. In this paper, the materials used for making phonon crystal are lowdensity polycarbonate fiber cotton and sound absorbing cotton in the well, which are stacked and embedded into 169 copper pipes with a radius of $0.5 \mathrm{~mm}$ at equal intervals. The noise frequency range selected by the experiment is $100 \mathrm{~Hz}$ to $1.5 \mathrm{kHz}$. Under the condition that the fixed position and other environmental conditions remain unchanged, a measurement point is taken at each interval of $100 \mathrm{~Hz}$, and the data measured at each frequency point are processed by matlab.

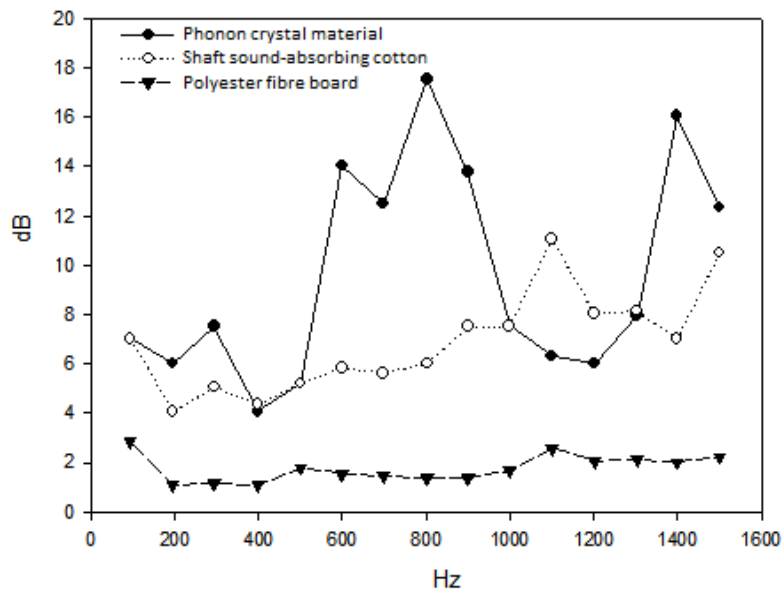

Figure 9: Noise reduction contrast of phonon crystal

After proving that phonon crystal material can play a good role in high frequency signal noise reduction, it begins to try to add phonon crystal material into active noise reduction system, combine active noise reduction technology with passive noise reduction technology, and conduct experimental comparison. The experimental results are shown in Figure 10.

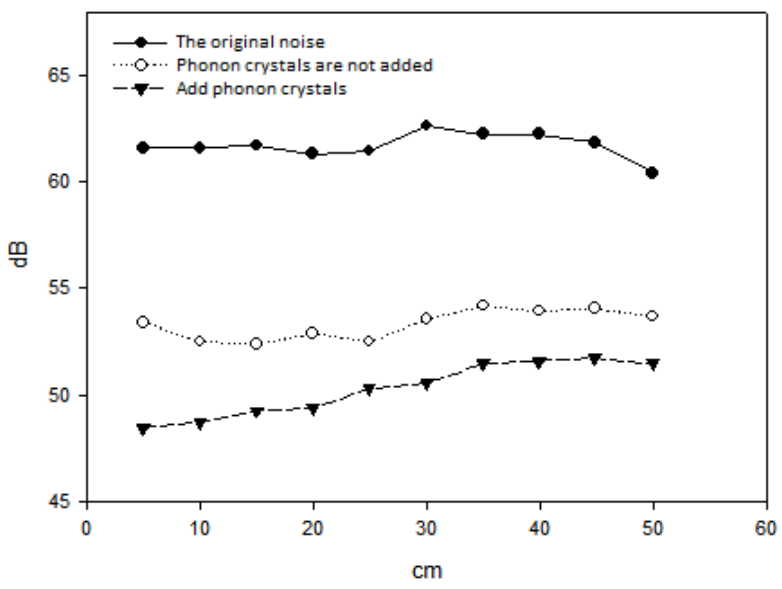

Figure 10: Experimental data after phonon crystal was added

It can be seen that the original noise is about $61.5 \mathrm{db}$. When active noise-reducing headphones without phonon crystal are used, the noise decibel value of the observation point is about $52-53 \mathrm{db}$, and the noise reduction amount is $8-9 \mathrm{db}$. When phonon crystal is added between secondary sound source and primary sound source, the noise decibel value after noise reduction is about $46-49 \mathrm{db}$, and the noise reduction amount is about $16 \mathrm{~dB}$, which has a good noise reduction effect.

\section{Conclusion}

This paper firstly analyzes the principle and system structure of adaptive active noise reduction, and clarifies the noise reduction structure of feedforward active noise reduction control system, feedback active noise reduction control system and front and feedback combined active noise reduction control system. Secondly, in order to implement the controller adaptive adjustment, achieve good noise reduction effect, to join the smaller mean square (LMS) algorithm, and based on the performance of the LMS feedforward adaptive active noise cancelling headphones were tested, by have good noise reduction effect in the low frequency signal, noise reduction effect, there is no advantage in the high frequency signal in the point of view; Finally, in view of the active noise reduction headphones can't play in the high frequency signal good noise reduction effect of this problem, blended in phononic crystal materials, and has carried on the experiment, the results after clear the blended in phononic crystal materials of active noise cancelling headphones can in the high frequency signal has a good noise reduction effect, to work on active headset noise reduction provides a new thought of optimization. 


\section{References}

[1] Tang Z M. "Current situation, harm and treatment of noise pollution," Ecological Economy, 2017,33(01):6-9.

[2] Yang J C, Wu X W, lv J H, et al. "Research on noise source measurement and prediction technology of uhv converter station," China Environmental Monitoring, 2018(1):138-144.

[3] Zhang M, Shang W N, Wang Y P, et al. "Characteristics and distribution rules of audible noise in uhv converter station," Architecture Science, 2017, 33(12):82-89.

[4] Shen D. "Research and application of noise control technology for distribution transformer," Shandong University of Technology, 2018.

[5] Zhang $\mathrm{P}$ J, Zhang L J, Meng D J, He Z. "Optimization method of loudspeaker and microphone placement in active control system of vehicle interior noise," Vibration and Impact, 2017,36(05):169-175.
[6] Yang P, Ying L M, Chen M, Wang J W, Wang G D. "Background noise processing method of ANC system secondary channel," Electrical Measurement and Instrumentation, 2017,54(08):99-103.

[7] Zangi K C. "A new two-sensor active noise cancellation algorithm," IEEE International Conference on Acoustics, Speech, and Signal Processing. IEEE, 1993, 2:351-354.

[8] Iwashita Y. "FEEDFORDWARD CONTROL UNIT FOR SERVOMOTOR: WO,WO/1990/011562," 1990.

[9] Kannan G, Milani A A, Panahi I M S, et al. "An Efficient Feedback Active Noise Control Algorithm Based on Reduced-Order Linear Predictive Modeling of fMRI AcousticNoise," IEEE Transactions on Biomedical Engineering, 2011, 58(12):3303-3309.

[10] Rafaely B, Jones M. “Combined feedbackfeedforward active noise-reducing headset--the effect of the acoustics on broadband performance," Journal of the Acoustical Society of America, 2002, 112(1):981-9. 